10 (2017) 4: 509-532

ISSN (print) 1689-5150

ISSN (online) 2450-7059

\title{
«Hanno incendiato tutti i santuari di Dio nel paese» (Sal 74,8b). Tempio, Nome e memoria nel Sal 74
}

\section{"They burned down every sacred shrine in the land" (Ps 74:8b). Temple, time and memory in Ps 74}

\section{"Spalili w kraju wszystkie miejsca świętych zgromadzeń" (Ps 74,8b). Świątynia, imię i pamięć w Ps 74}

\begin{abstract}
Psalm 74 is a communal lament over the destruction of the temple, an event that for the worshipper(s) signifies a "loss of the centre," a destabilisation of the perception of space and time. In Ps 74, these two dimensions are strictly connected to temple (space) and memory (time). The article tries to analyse the interaction of the abovementioned elements in order to detect how they contribute to the depiction of such a destabilisation. After a short discussion about the structure and the logic of the psalm, the verses in which memory and the temple are mentioned (vv. 2-8.18-23) are closely analyzed and a concise conclusion is then drawn. The worshipper(s) feel themselves in a situation of eternal present and without a centre, forgotten by God, since the distress seems to endure without ending. The memory of the covenant (v. 2) and of the victory of God over the primeval chaos at the beginning of creation (vv. 12-17) opens a possibility of hoping for a future re-stabilisation of time and space, even if in an undetermined way (v. 21).
\end{abstract}

Streszczenie. Psalm 74 jest zbiorowym lamentem nad zniszczeniem świątyni, wydarzeniem, które dla czciciela (czcicieli) oznacza "utratę centrum", destabilizację postrzegania przestrzeni i czasu. Te dwa wymiary są ściśle związane w Psalmie 74 z świątynią (przestrzenia) i pamięcią (czasem). Artykuł podejmuje próbę analizy interakcji wyżej wspomnianych elementów, aby odkryć jak przyczyniają się one do przedstawienia tej destabilizacji. Po krótkiej dyskusji struktury i logiki psalmu, następuje dokładna analiza wersetów, w których wspomniana jest pamięć i świątynia (wersety 2-8.18-23). Artykuł zwieńczony jest krótkim podsumowaniem. W sytuacji wiecznej teraźniejszości czciciel (czciciele) odczuwają, że zostali pozbawieni centrum, zapomniani przez Boga, gdyż ich niedola wydaje się być bez końca. Pamięć przymierza (w. 2) i zwycięstwa Boga nad pierwotnym chaosem na początku aktu stworzenia (wersety 12-17) otwiera możli- 
wość nadziei na powrót do przyszłej stabilizacji czasu i przestrzeni, nawet jeśli miałoby to nastąpić w bliżej niekreślony sposób (w. 21).

Keywords: Ps 74; Psalms of Asaph; Third Book of the Psalter; memory; spatial studies; temple; name of God.

Słowa klucze: Psalm 74; Psalmy Asafa; trzecia Księga Psałterza; pamięć; studia przestrzeni; świątynia; imię Boga.

\section{Introduzione}

Cion e il tempio costituiscono, in particolare nel Salterio, una sorta di «centro $\checkmark$ simbolico», nel quale la presenza di Dio trova una sua concretizzazione spaziale e temporale ${ }^{1}$. All'interno del Salterio, tuttavia, non si trovano solo celebrazioni della preminenza e della «centralità» di Sion (cf ad es. Sal 46; 48; 84; 87; ecc.) ma anche lamenti per la distruzione del tempio ${ }^{2}$. Tra questi ultimi, il Sal 74 - insieme con il Sal 79 - spicca per la vividezza con cui tale catastrofe viene rappresentata e, in qualche modo, «lamentata».

La perdita del tempio ha, in qualche modo, a che fare con una sorta di «perdita del centro», di una destabilizzazione della percezione stessa dello spazio e del tempo. Questi due assi fondamentali sono essenzialmente collegati, nel Sal 74, alla realtà stessa del tempio (spazio) e a quella della memoria (tempo), la cui interazione permette di cogliere come il testo rappresenta e affronta, in un contesto di preghiera, tale "perdita del centro». Nel contributo che segue ci proponiamo di analizzare l'interazione tra i due elementi succitati, in modo da mettere in luce la drammatica interna del salmo e la modalità con cui spazio e tempo vi vengono rappresentati. In questo modo, sarà forse possibile cogliere meglio un elemento essenziale della drammatica del Sal 74 e, più in generale, della funzione che la memoria vi svolge ${ }^{3}$.

1 Tra l'ampia letteratura in proposito, cf.: Hartenstein, Unzugänglichkeit; Zenger, «Das Mythische», 233-251; idem, «Semiotiserung», 180-206; Janowski, «Die heilige Wohnung», 24-68; Körting, Zion; Janowski, «Der Ort», 369-397. Sul tempio come «centro simbolico» della Bibbia Ebraica, cf. anche Fishbane, «Center», 6-27.

2 Questo genere di lamenti si trovano anche fuori dal Salterio. Cf. ad es. Lam 2. Sul tema, cf. Engle, Psalm 74, 214-237.

3 Per la letteratura sul Sal 74, oltre ai commentari, cf. in particolare: Donner, «Argumente», 41-50; Weiss, «Methode», 88-112; Ploeg, «Psalm 74», 204-210; Emerton, «Translation», 120-121; Loretz, Leberschau; Engle, Psalm 74; Spieckermann, Heilsgegenwart, 122-133; Human, «Berit», 57-66; Bouzard, Communal; Emmendörfer, Gott, 77-102; Boadt, «Panels», 533-550; Basson, ««Only Ruins Remains». Psalm 74 as a Case of Mundus Inver- 
Dopo una prima discussione della struttura e della logica interna del salmo, ci occuperemo della rappresentazione della memoria e del tempio, per poi trarre, nel paragrafo finale, alcune conclusioni ${ }^{4}$.

\section{Struttura e logica del Sal 74}

Sebbene gli studiosi non concordino nel determinare la struttura del Sal 74, non di meno si può registrare un accordo quasi unanime su alcuni punti ${ }^{5}$. Il testo è articolato in 23 verselines e 48 cola $^{6}$ e una prima considerazione degli indicatori di struttura più significativi permette di discernerne in filigrana la strutturazione di fondo.

Vanno notati, innanzitutto, alcuni blocchi caratterizzati dall'uniformiצריר צà quanto a soggetto verbale, in particolare i vv. 4-8 (verbi con soggetto [v. 4a]; cf anche אויב [v. 3b]) $)^{7}$ e i vv. 13-17 (i verbi ${ }^{8}$ hanno tutti come soggetto Dio; cf anche la ripetizione per 7x del pronome אתה ai vv. 13a.14a.15ab.16b.17ab) ${ }^{9}$. I vv. 2-3.18-23, per contro, si caratterizzano per l'uso insistito degli imperativi di $2^{\text {a }}$ singolare, sia positivi (vv. 2.3.18a.20a.22) che negativi (vv. 19.21a.23a) ${ }^{10} \mathrm{e}$,

sus», 128-137; Baumann, «Psalm 74», 91-103; Venter, «Psalm 74», 533-545; Lippke, «Ps 74:15», 141-152; Tsumura, «The Creation Motif», 547-555; Greene, «Destruction», 85-101.

4 Questo studio amplia quanto già affermato in Pavan, «He Remembered».

5 Per una rassegna delle posizioni, oltre ai commentari, cf. Emmendörfer, Gott, 82; Lugt, Cantos II, 313-314. Cf. anche Basson, Metaphors, 210-219; Goldingay, Psalms 2, 423; Gärtner, Geschichtspsalmen, 116-117; Tsumura, «The Creation Motif», 547-555; Greene, «Destruction», 85-101.

6 Lugt, Cantos II, 313, da cui riprendiamo anche la terminologia (colon, cola, bicola, tricola), anche se su questo punto non cè unanimità tra gli studiosi: cf. le posizioni enumerate da Idem, Cantos II, 313-314, a cui va aggiunto Weber, Werkbuch Psalmen III, 27-28. In generale, comunque, gli studiosi concordano nel considerare tricola i vv. 2.9.

7 In questi versetti sono «condensate» le menzioni del tempio nel Sal 74. I vv. 5.6, tuttavia, costituiscono una nota crux interpretum e sono lasciati non tradotti da alcuni commentatori (cf. ad es. Spieckermann, Heilsgegenwart, 123). Per le posizioni e le proposte degli studiosi in proposito, cf. Emerton, «Notes», 374-377; Robinson, «Solution», 120-121; Sollamo, «The Simile», 178-187; Tate, Psalms, 242; Emmendörfer, Gott, 79-80; Hossfeld Zenger, Psalmen 51-100, 357-358.

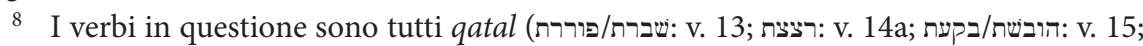
הכינות: v. 16b; יצרתם/הצבת: v. 17), con la sola eccezione dell'yiqtol al v. 14b (תתננ). Il v. 16a è una frase nomninale.

9 Cf. Tsumura, «The Creation Motif», 547-555; Greene, «Destruction», 85-101.

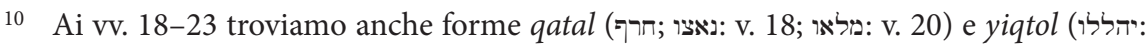
v. 21) e participio (עלה: v. 23). Sullalternanza tra imperativi positivi e negativi, cf. anche Weber, Werkbuch Psalmen III, 27. 
quindi, per la preminenza del soggetto divino ${ }^{11}$. In generale, allinterno di un salmo in cui domina, nella direzione del discorso, il riferimento in «tu» a Dio, spicca il v. 12, l'unico in cui si parla di Dio in $3^{\text {a }}$ persona ${ }^{12}$.

Oltre a questa "condensazione» di blocchi di versetti in base alle forme verbali, si segnalano anche alcuni fenomeni di ripetizione abbastanza significativi, a diversi livelli. Innanzitutto, spicca la presenza di domande retoriche

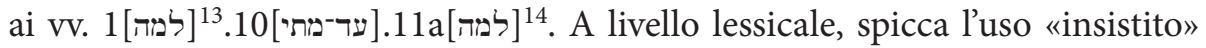

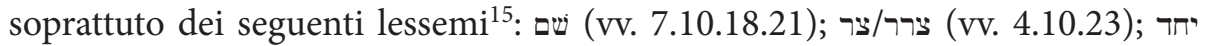

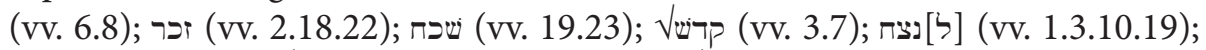

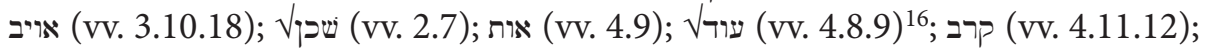
קדם (vv. 2.12); ארים (vv. 7.8.12.17.20); ארץ (vv. 10.17.18.22; cf anche v. 17b cf anche: אלהים (vv. 1.10.12.22), יהוה (v. 18) e אל (v. 8). Queste ricorrenze evidenziano le corrispondenze tra alcuni versetti (vv. 10.18 in particolare: שם ; נאץ ; שרף) e la presenza di lessemi che, in un certo senso, "percorrono» tutto il salmo (in

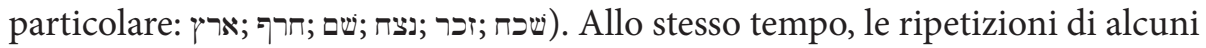
lessemi tendono a «compattare» i blocchi di versetti sopra evidenziati: $\mathrm{cf}$ ad es. אות per i vv. 4-9; אתה per i vv. 13-17; שכת e שכר per i vv. 18-23

Se, quindi, il salmo si caratterizza per la simultanea presenza di blocchi di versetti abbastanza riconoscibili (vv. 2-3/4-8/13-17/18-23), da una parte e una serie di versetti tra loro simili ma «indipendenti» (vv. 1.10.11a; $\mathrm{cf}$ anche v. 12), dall'altra, non è però del tutto chiara la suddivisione interna di questo materiale ${ }^{19}$. Proprio in relazione a questi versetti «indipendenti», infatti, ruota-

11 In questi versetti, infatti, si ritrovano anche i poveri (v. 21) e il «fragore» dei nemici (v. 23b), oltre ai «confini della terra» (מחשכי־ארץ: v. 20b).

12 Lugt, Cantos II, 313. Cf. però anche il v. 8b. L'insistenza sul «tu» divino si ritrova anche nell'uso ampio del prefisso di $2^{\text {a }}$ persona singolare (vv. 1-4.7.10-11.13.16.18-19.21-23).

13 Sull'uso di questa particella interrogativa, cf. Emmendörfer, Gott, 82-83.

14 Sui problemi testuali implicati nel v. 11b, cf. Castellino, Salmi, 309.883; Tate, Psalms, 243; Hossfeld - Zenger, Psalmen 51-100, 358; Gerstenberger, Psalms 2, 78.

15 Cf. Lugt, Cantos II, 315-316, per una lista più esaustiva.

16 A questo proposito, cf. anche i fenomeni di acrostichia e allitterazione segnalati da Weber, Werkbuch Psalmen III, 27. Cf. anche Human, «Berit», 57-66.

17 In questo versetto il nome חרו suona come omofono - e forse un gioco di parole della radice חרף, anche se di etimologia probabilmente differente (חרף II: "passare l'inverno»; cf. Is 18,6; Sir 43,16): cf. Clines, Dictionary III, 320.

18 Un elenco più dettagliato delle corrispondenze lessicali del salmo in Lugt, Cantos II, 313-314.

19 Cf. la diversità di interpretazioni del 1 ai vv. 6.12: idem, Cantos II, 315-316. 
no le proposte di struttura formulate dagli studiosi, riducibili sostanzialmente a due ${ }^{20}$ :

1. da una parte, alcuni autori vedono nel salmo una scansione fondamentalmente tripartita $(1-11.12-17.18-23)^{21}$. Tale suddivisione si basa sulle osservazioni sopra raccolte e su una duplice constatazione: l'inclusione formata dai vv. 1 e $10.11 \mathrm{a}$; la differenza radicale tra il v. 11 e il v. $12^{22}$;

2. dall'altra, un altro gruppo di autori divide ugualmente il salmo in tre grandi «blocchi» ma con diversa scansione (1-9.10-17.18-23 ${ }^{23}$. Questa diversa distribuzione del materiale viene argomentata a partire da due osservazioni: da una parte, i vv. 1 e 10.11a costituiscono una sorta di «ritornello» con cui iniziano i primi due «blocchi» del salmo; dall'altra, i vv. 10 e 18 condividono - come sopra evidenziato - alcuni importanti vocaboli. Queste osservazioni tendono a far considerare i vv. $1.10 .11 \mathrm{a}$ e 18 come l'inizio delle tre grandi parti fondamentali del salmo ${ }^{24}$.

A far da sostegno all'una o all'altra ipotesi viene portata, quindi, dagli autori tra gli altri una diversa valutazione dei legami lessematici e della sintassi. Allo stesso modo, anche considerazioni di ordine diacronico influiscono sulla formulazione della struttura del testo ${ }^{25}$.

20 Data la diversità della terminologia adottata dagli autori, preferiamo usare una denominazione piuttosto generica («blocchi») al posto di una terminologia più specifica (stanza; strofa; canto). Cf. la discussione in idem, Cantos, 69-92.

21 Cf. tra gli altri Emmendörfer, Gott, 77-82; Cole, Shape, 30-33; Hossfeld - Zenger, Psalmen 51-100, 358-360. Per Boadt, «Panels», 540-541, la struttura sarebbe concentrica (1a.1b-11.12.13-23). Tra le proposte recenti, cf. Tsumura, «The Creation Motif», 547-55; Greene, «Destruction», 85-101.

22 Questa «cesura» è riconosciuta dalla maggioranza degli autori: cf. Lugt, Cantos II, 313-314.

23 Così, tra gli altri, van der Lugt, Girard, Pannier-Renard, Briggs (cf. l'enumerazione in idem, Cantos II, 313-314). Cf. anche la struttura bipartita di Weber, Werkbuch Psalmen III, 2627 (1-11//12-23, due parti a loro volta articolare al loro interno in 1-5.6-11//12-17.18-23).

24 Cf. la diversa lettura di idem, Werkbuch Psalmen III, 26-27, che pure nota la corrispondenza tra vv. 10 e 18.

25 Cf. Spieckermann, Heilsgegenwart, 126-128; Emmendörfer, Gott, 96-97; Hossfeld Zenger, Psalmen 51-100, 360-363; Weber, Werkbuch Psalmen III, 26. Il problema della datazione del testo (e, quindi, dell'identificazione del tempio di cui si lamenta la distruzione) vede gli autori separati in due gruppi (per una rassegna, cf. Emmendörfer, Gott, 82): quelli che sostengono una datazione nell'immediato postesilio; quelli che situano la stesura del salmo in epoca maccabaica. Singolare la posizione di Weber, Werkbuch Psalmen III, 26: il salmo sarebbe da collegare con la caduta di Samaria (722 a.C.). Per ulteriori ipotesi, cf. 
In ultima analisi, la seconda ipotesi appare, per molti versi, quella che maggiormente rivela la dinamica interna del salmo. Particolarmente rilevante, infatti, per l'articolazione drammatica del testo e per il dispiegarsi del suo messaggio appare la corrispondenza - quasi un ritornello ${ }^{26}$ - tra le parti iniziali dei tre blocchi fondamentali ${ }^{27}$ :

\begin{tabular}{|c|c|c|c|}
\hline & $1-3$ & $10-12^{*}$ & 18-19 \\
\hline לנצח & v. 1 & v. 10 & v. 19 \\
\hline אויב & v. 3 & v. 10 & v. 18 \\
\hline אלהים & v. 1 & v. 10 & v. 18 \\
\hline שמך & & v. 10 & v. 18 \\
\hline נאץ & & v. 10 & v. 18 \\
\hline חרף & & v. 10 & v. 18 \\
\hline זכר & v. 2 & & v. 18 \\
\hline למה & v. 1 & v. $11^{* *}$ & \\
\hline
\end{tabular}

* Cf. le ragioni in idem, Cantos II, 316-317.

** Cf. anche l'interrogativo ער־מת al v. 10. Funzione analoga avrebbe il vocativo יהוה al v. 18a: cf. idem, Cantos II, 312.

Sostanzialmente, queste corrispondenze - e altre elencate dagli autori ${ }^{28}$ - si comprendono meglio come ritornello, nella prospettiva di una struttura alternata, che come inclusione (tra i vv. 1 e 10.11) ${ }^{29}$. In questo modo, lo sviluppo dei tre blocchi potrebbe essere sintetizzato come segue:

1. nel primo (vv. 1-9) viene articolata un'introduzione (vv. 1-3a) a cui segue la descrizione dell'azione distruttrice dei nemici (vv. 3b-8) $)^{30}$, conclusa

anche Schökel - Carniti, Salmi II, 32-33. Allo stesso modo, gli autori tendono a considerare alcuni parti del salmo secondarie, principalmente i vv. 2c.9b.19-21 (cf. Hossfeld - Zenger, Psalmen 51-100, 360-363).

26 Questo è, in effetti, il termine usato da Lugt, Cantos II, 315-317.

27 La divisione dei tre blocchi: cf. idem, Cantos II, 315-316. Lautore vede l'unione, in questi passaggi, di lamento e preghiera, se si tiene - con il TM - l'imperativo al v. 11b.

28 Cf. anche Auffret, «Psaume LXXIV», 129-148; idem, ««Souviens-toi de ton assemblée!». Étude structurelle du Psaume 74», 21-31.

29 Struttura alternata in Lugt, Cantos II, 313-315.

30 La descrizione viene introdotta al v. $3 \mathrm{~b}$ da un improvviso cambio di soggetto e di tempo verbale (Dio in 3a [imperativo] e il nemico in 3b [qatal]). La descrizione si snoda 
con una sorta di dolorosa constatazione della situazione attuale (v. 9) ${ }^{31}$. La parte introduttiva è costituita sostanzialmente da un lamento (למה: v. 1) e da due imperativi (זכר: v. 2a; הרימה: v. 3a) al primo dei quali è collegato un riferimento alle origini dell'alleanza tra Dio e Israele (vv. 2bc); 2.nel secondo (vv. 10-17), dopo un'introduzione in forma di domande retoriche (vv. 10.11) ${ }^{32}$, viene articolato un inno in cui si celebra il dominio divino manifestato alle origini ( $c f$ קדa: v. 12a) del mondo, espresso sostanzialmente come uno «spezzare» le forze primordiali personificate dai תנינים (v. 13b) e dal לויתן (v. 14a) e un "ordinare» spazio e tempo (vv. 15-17) $)^{33}$;

3. nel terzo (vv. 18-23) si nota, come detto, una struttura ordinata a partire dal ripetersi di imperativi positivi e negativi ${ }^{34}$. A Dio viene nuovamente rivolto l'invito a ricordare (זכר) l'insulto dei nemici (vv. 18.22b), a non di-

attorno all'immagine della profanazione (v. 4), del «disboscare» (vv. 5-6) e del dare alla fiamme (vv. 7-8), con una conclusione finale (v. 9). I vv. 4.9 sono collegati dal sostantivo אות e costituiscono una sorta di «introduzione» e «conclusione». Le ricorrenze dei termini legati al tempio (משכן ;מקרש ; מוער) costituiscono una sorta di filo rosso in questa trama. Cf. idem, Cantos II, 313-315, per differenti proposte.

31 La funzione conclusiva del v. 9 è sottolineata dall'uso del tricolon e dall'emergere del «noi», spettatore e narratore della distruzione dei nemici.

32 I due versetti contengono, in qualche mode, una reduplicazione della domanda retorica, la prima focalizzata sui «nemici» (v. 10) e la seconda su Dio (v. 11). In questo modo, il contrasto fondamentale del salmo (silenzio/ira di Dio e dilagare dei nemici) è come sintetizzato: cf. ancora Lugt, Cantos II, 315.

33 Cf. Emmendörfer, Gott, 92-96. Sulla funzione di questi versetti: Broyles, Conflict, 151.153. Sulla loro struttura, cf. anche Auffret, «Psaume LXXIV», 134-136. Cf. anche Brown, Seeing, 143.189; Basson, Metaphors, 215-216. A livello di sostantivi, i vv. 13-17 sembrano disporsi così:
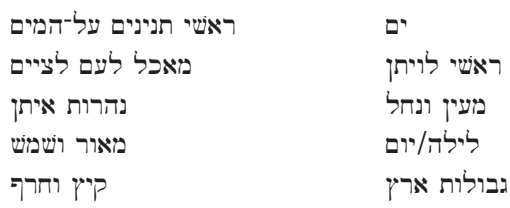

La disposizione sembrerebbe seguire uno schema incentrato su due grandi ambiti: mare (vv. 13-15) e alternarsi del tempo (vv. 16-17; cf. però: גבולות ארץ). Questi versetti contengono riferimenti chiari non solo a testi del VOA di natura «mitica» ma anche a Es 15 (cf. Spieckermann, Heilsgegenwart, 128-131; Greene, «Destruction», 90-99). I verbi sono, per contro, da una parte legati al «distruggere» (הובשת/בקעת/רצצת/תברת/פוררת: vv. 13-15; cf. anche תתנגו al v. 14b) e dall'altra allo «stabilire» (צרתם/הצבת/הכינות/פרתות: vv. 16-17).

34 Cf. Weber, Werkbuch Psalmen III, 26. 
menticare (אל-תשכת) la vita dei suoi poveri (v. 19b) e il tumulto dei nemici (v. 23a), a guardare all'alleanza (הבט לברית: v. 20a) e a rispondere all'insulto dei nemici che lo chiamano a contesa (ריבה ריבך/קומה: v. 22a)

Lungi dall'apparire una struttura statica o senza movimento ${ }^{36}$, l'articolazione del salmo presuppone, in qualche modo, una sorta di «processo» interno di pensiero e, allo stesso tempo, un sottile slittamento dei termini iniziali del «problema» posto ex abrupto dal v. 1.

Per tentate di cogliere, nei suoi tratti essenziali, la logica interna del salmo, è forse utile coniugare i dati fin qui raccolti con una breve considerazione della sua struttura comunicativa ${ }^{37}$. Concretamente, si tratta soprattutto di identificare il locutore del salmo e il modo in cui vengono rappresentati $i$ "protagonisti» principali dell'azione, in modo tale da evidenziare la dinamica del discorso dispiegato dagli oranti e, quindi, per l'appunto, la sua logica.

Il primo punto - l'identificazione del locutore del salmo - pone immediatamente dei problemi. In proposito si possono, infatti, reperire delle indicazioni apparentemente contrastanti ${ }^{38}$ :

1. al v. 9 compare per 2x un suffisso di $1^{a}$ persona plurale (לא-אתנו ; אותתינו) e un verbo della stessa persona (ראינו);

2. al v. 12, compare un improvviso suffisso di $1^{\text {a }}$ persona singolare (מלכיבי);

35 Nella fitta trama di imperativi, spiccano il v. 20b (proposizione esplicativa introdotta da כי), il v. 21 (formulato in termini di «auspicio»: due jussivi) e il v. $23 \mathrm{~b}$ (con un participio: עלה תמיד

36 Questo il giudizio di Girard, Les Psaumes redécouverts, 298.

$37 \mathrm{Su}$ questo genere di approccio al testo salmico, cf. Wagner, «Dichten», 271-294; idem, Beten und Bekennen; idem, «Strukturen», 197-216; Petrany, Pedagogy.

38 Il problema centrale di queste indicazioni è l'apparente contraddizione tra «io» (v. 12a) e «noi» (v. 9). Di importanza centrale è la designazione "לאס nel titolo, che va probabilmente intesa come indicazione di una «voce» singola che parla a nome del popolo: cf. l'analisi più generale (per il III libro) della questione in Pavan, «He Remembered», 195199. Nelle pagine che seguono ci riferiremo sempre all'orante (al singolare), lasciando aperta la questione.

39 La LXX ( $\beta \alpha \sigma \iota \lambda \epsilon \dot{\jmath} \varsigma \dot{\eta} \mu \omega \hat{\omega} \nu)$ e la Vulgata (rex noster) leggono: «il nostro re». Girolamo (rex meus) segue invece il TM. 
3. ai vv. 1-3, in modo indiretto, i locutori del salmo si autodesignano come Israele, popolo dell'alleanza, utilizzando per fare ciò una terminologia «tipica»: עאן מרעיתך (v. 1b); ערתך (v. 2a); שבט נחלתך (v. 2b)

4. ai vv. 18-23, per contro, il soggetto umano di riferimento sembrano essere piuttosto «i poveri» ${ }^{41}$ : cf ענייך (v. 19b) רך (v. 21a); עני ואביון (v. 21b)

Se l'identificazione esatta del locutore del salmo sembra, in qualche modo, ostacolata da queste oscillazioni, i nemici e Dio - gli altri due protagonisti del salmo - vengono rappresentati in modo abbastanza «regolare». Per quanto riguarda il «personaggio divino» ${ }^{44}$, si può sottolineare, tra l'altro, come:

1. ai vv. 1-3a, il Signore appare nella sua «ira» (יעשן אפך "ינחת: v. 1) ma anche come pastore del gregge (צאן מרעיתך: v. 1b), «possessore» di Israele (ערתך קנית קדם:v. 2a), che ha riscattato come sua eredità (גאלת שבט נלחתך: v. 2b) e «abitatore» del monte Sion (הר־ציון זה שכנת בו: v. 2c)

2. al v. 11, Dio viene di nuovo rappresentato come adirato, come «inerte» (תשיב ידך וימימנך: v. 11a) o rivolto contro il popolo (כלה: v. 11b)

3. ai vv. 12-17 la regalità (מלכי: v. 12a) di Dio viene celebrata nel suo dominio sulle forze cosmiche primordiali;

4. ai vv. 18-23, infine, l'insistito uso degli imperativi sottolinea ancora una volta la situazione di «inattività» divina - dovuta all'ira, a giudicare dal v. 1 - di fronte ai nemici (cf v. 23) ${ }^{47}$;

5. bisogna anche segnalare anche come ai vv. 10.18.22.23 Dio appare come

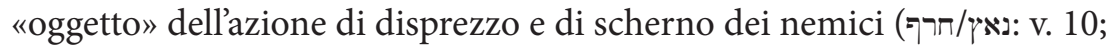

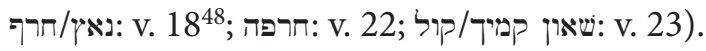

40 Correttamente, Emmendörfer, Gott, 81, nota che שבט נחלתך va inteso come stato costrutto e non come giustapposizione dei due oggetti di גלתלך.

41 Cf. Hossfeld - Zenger, Psalmen 51-100, 360-361.

42 In questo senso andrebbe letta anche l'espressione נפש תורך al v. 19a: non tanto, cioé, come un riferimento a Israele ma ai poveri, in forza del parallelismo con il v. 19b. Cf. Begg, «Dove», 78-81.

43 Tra il v. $19 b$ e il v. 21 cè un'alternanza tra singolare e plurale.

$44 \mathrm{Su}$ questo punto, cf. Basson, Metaphors.

45 Pavan, «He Remembered», 202-203.

46 Anche il v. $11 \mathrm{~b}$ costituisce, come già notato, una nota crux interpretum. Le versioni antiche oscillano: cf. Tate, Psalms, 243 di cui adottiamo la lettura («[Draw it] from the midst of your bosom; end it!»). Oltre a quelle già menzionate, cf. anche le ipotesi elencate in Hakham, Psalms, 17.

47 Il v. 20b può contenere un'allusione all'attività dei nemici.

48 Questo è l'unico versetto in cui appare il tetragramma divino. 
La menzione dei nemici percorre, in qualche modo, tutto il salmo (vv. 3b8.18.22.23; $\mathrm{cf}$ anche vv. $20 \mathrm{~b})^{49}$. A questo proposito possiamo sottolineare:

1. l'uso costante di alcuni lessemi per designare i nemici: אויב (vv. 3.10.18); צרר/צר (vv. 4.10.23). Cf anche: עם נבל (v. 18b); קמיך (v. 23b);

2. la descrizione dell'azione distruttutiva dei nemici ai vv. 3b-8;

3. la citazione diretta delle parole dei nemici (v. 8a: נינם יחד

4. infine, come già evidenziato, il fatto che la distruzione del tempio operata dai nemici equivale ad un «insulto» rivolto direttamente a Dio (cf vv. 10.18.22.23). Bisogna notare che solamente al v. 23, in conclusione del salmo, i nemici vengono qualificati con un suffisso di $2^{\text {a }}$ singolare (צריך; קמיך; cf anche v. 22: חרפתך ;).

Il complesso intreccio creato dall'interrelazione dei tre personaggi permette di determinare con maggior precisione lo sviluppo del salmo. Da questo punto di vista, due sono gli elementi che spiccano in modo particolare: da una parte la comprensenza di due generi di affermazioni, l'una che attribuisce all'ira divina la causa della sventura presente (vv. 1.11) e l'altra che la colloca nei nemici, essi stessi oltraggiatori del Nome divino (vv. 10.18); dall'altra, la già menzionata «alternanza» tra i riferimenti più generali ad Israele (v. 2) e quelli, più specifici, ai poveri (vv. 19b.21). I nemici, dal canto loro, vengono rappresentati, per così dire, dall'esterno, in modo storicamente indeterminato, non entrando che come «fattore esterno» nella relazione tra Dio e Israele - enfatizzata, quest'ultima, in modo particolare ${ }^{51}$.

In ultima analisi, il salmo appare imperniato sulla richiesta di un intervento divino (cf vv. 1-3a.18-23; cf anche v. 11) di fronte alla distruzione del santuario (vv. 3b-10). Tale richiesta si snoda sul filo di una logica stringente: dopo aver presentato il «problema» e la prima richiesta (v. 1$)^{52}$, lorante richiama prima la memoria del passato fondativo dell'alleanza tra Dio e Israele, sottolineando con forza gli impegni del primo verso il secondo. Dopo la descrizione della cata-

49 Cf. Pavan, «Nemico», 273-298.

50 Weber, «Direkte Rede», 191-267. Ai vv. 4-8 ci sarebbe un focus sul «rumore» prodotto dai nemici (grida, urla, ecc.) oltre che sul danno materiale da loro procurato: cf. Hakham, Psalms, 16-17.

51 In altre parole, l'asse comunicativo del salmo è imperniato sulla relazione «io-tu» tra Dio e Israele, come si rivela, ad es., dall'uso insistito dei suffissi di $2^{\text {a }}$ singolare. Il perno «narrativo» (eccetto il v. 8a) è, chiaramente, il locutore del salmo.

52 Spieckermann, Heilsgegenwart, 127. 
strofe dell'irruzione dei nemici nel tempio (vv. 3b-9) - essa stessa una richiesta implicita $^{53}$-, viene rinnovata la richiesta dell'intervento divino e la cessazione dell'ira (vv. 10-11). A questo punto, in netto contrasto con quanto riportato ai vv. 3b-9 e 10.11 ( $\mathrm{cf}$ il, avversativo al v. 12a), viene celebrata l'azione creatrice di Dio alle origini del creato, azione che rivela la sua regalità universale (vv. 13-17). Questa «parentesi» centrale all'interno del salmo ${ }^{54}$ introduce la serie di richieste dei vv. 18-23: poiché il Signore ha rivelato la sua regalità come signoria sulle forze primordiali del creato (vv. 13-15) e come imposizione di ordine e confini allo stesso (vv. 16-17), così ora deve guardare all'azione distruttrice dei nemici (vv. 18.19a.20b.22.23) e porvi rimedio. Beneficiari dell'azione implorata sono, questa volta, i poveri (vv. 19b.21), termine con cui probabilmente l'orante si identifica e con cui l'identità o la condizione di Israele viene meglio specificata. Il salmo si chiude in sospeso (vv. 22-23), con l'invito rivolto a Dio perché «ricordi» l'oltraggio quotidiano del nemico (כל־ה: v. 22b), una sorta di «fragore» che sale continuamente (תמיד: v. 23b).

In ultima analisi, la struttura del salmo sembra implicare, allo stesso tempo, una sorta di movimento «tesi-antitesi-sintesi» ${ }^{55}$ e di climax, in cui le prime due parti (costruite a loro volta anche sul contrasto tra distruzione dei nemici e regalità divina ${ }^{56}$ ) preparano la richiesta finale, il cui culmine è la designazione dei nemici - per la prima volta nel salmo - come nemici di Dio (cf vv. 4.10.18.22.23) ${ }^{57}$.

\section{Memoria e tempio nel Sal 74}

Nella struttura del salmo, il tema della memoria svolge una funzione non secondaria, come appare innanzitutto dalle già menzionate occorrenze esplicite dei lessemi שכח (vv. 2.18.22) (19.23). Lo stesso si può dire del tempio

53 Cf. Schökel - Carniti, Salmi II, 37-40.

54 Cf. Janowski, «Doppelgesicht», 89-97.

55 Ad es. nel passare dal lamento per l'ira di Dio (vv. 1.11) a quello per l'azione distruttrice dei nemici (vv. 10.18) nelle sezioni introduttive delle tre parti.

56 Si nota un contrasto, chiaramente, anche tra il v. 2 e i vv. 3-9. Cf. Weiss, «Methode», 102-106.

57 Lugt, Cantos II, 318. Cf. anche Wälchli, Zorn, 58-59. Secondo Hossfeld - Zenger, Psalmen 51-100, 360-361, il tema della lotta di Dio contro i suoi nemici e quello della minaccia ai poveri si sovrappongono nel salmo. Cf. anche la scansione individuata da Emmendörfer, Gott, 81-82 (Klage-Hymnus-Bitte). Secondo Willesen, «Psalm LXXIV», 299306, l'origine del Sal 74 sarebbe del tutto cultuale. Cf. anche Broyles, Conflict, 151; Bouzard, Communal, 147-151. 
(cf vv. 3b-8), la cui distruzione è la causa fondamentale del lamento. Nelle righe che seguono tenteremo di vedere come questi due aspetti interagiscono nel definire la struttura spaziale e temporale del testo e, quindi, la sua unità drammatica.

\subsection{I lessemi שכת e זכר}

Ilessemi שכח זכר ricorrono nella parte iniziale (v.2a) e finale (vv.18a.19b.22b.23a) del salmo, quasi a costruire una sorta di inclusione generale ${ }^{58}$. Al v. 2, il verbo זכר è costruito in modo transitivo, avendo come oggetto ערתך e, di seguito, la rievocazione dell'«atto fondatore» di Israele da parte di Dio. Allo stesso tempo, i vv. 18-23 appaiono strutturati fondamentalmente su queste ricorrenze, in due composizioni chiastiche all'inizio e alla fine di questo blocco ${ }^{59}$ :

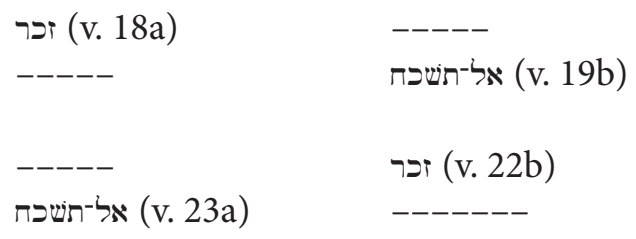

I verbi ricorrono sempre con soggetto divino e sempre all'imperativo singolare ed esprimono, pertanto, in qualche modo, l'orientamento fondamentale della richiesta degli oranti. Oggetto dei verbi risultano essere: עדתך (v. 2a); זמרת (v. 18a) chiede a Dio di muovere la sua «memoria» o verso Israele o verso il disprezzo dei nemici, il perdurante affronto che questi fanno al Signore stesso e al suo nome.

L'insistenza degli imperativi legati all'ambito della memoria sottolinea, per differenza, il perdurare dell'oblio di Dio, qualificato essenzialmente come un «non agire» in favore del popolo (cf. v. 11) o anche come un «rigettarlo»

58 Riprendiamo e ampliamo quanto già scritto in Pavan, «He Remembered», 202-206.

59 Lugt, Cantos II, 317, parla in proposito di parallelismus stropharum. Nella sua divisione (18-19.20-21.22-23), i vv. 20-21 costituirebbe il centro di questo blocco. Secondo Greene, «Destruction», 89, il v. 21 (עני ואביון יהללו שמך) costituirebbe il centro della struttura chiastica dei vv. 19-23.

60 Dio è invitato a ricordare זמאת, «questa cosa» (cf. JM $\$ 143 a$ ), vale a dire: l'insulto

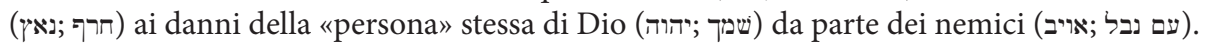
Cf. anche Dahood, Psalms II, 207; Broyles, Conflict, 153; Spieckermann, Heilsgegenwart, 128-129; Basson, Metaphors, 217-218. 
(cf v. 1) ${ }^{61}$. Questa accezione emerge, in particolare, considerando i verbi posti in parallelo con שכת e שכר ai vv. 18-23: cf in particolare אל־תן (v. 19a); ריבה/קומה (v. 22a). Tale oblio fa, per così dire, da sfondo alla preghiera dellorante e costituisce il problema che perdura. Da questo punto di vista, il salmo si si colloca in una sorta di "presente indeterminato", in cui la situazione di rovina sembra estendersi in modo indefinito (cf vv. 9.22b.23). Proprio sul senso della «durata» si appoggia, per così dire, la richiesta dellorante (cf vv. 9.10; cf לנצח ai vv. 1a.10b.19b), che arriva, per questo, a definire «eterne» le rovine del tempio (משאות נצח: v. 3a) su cui Dio deve muovere i suoi passi ${ }^{62}$.

Il ricorso ai verbi collegati alla memoria e, quindi, l'appello alla «memoria divina» diventa, così, un appello a «sciogliere» una situazione di oblio che perdura, il cui effetto è il dilagare dell'azione di distruzione e di oltraggio dei nemici (cf vv. 10.18.22.23). Tale memoria viene, per così dire, orientata verso alcuni punti precisi, più o meno così identificabiliti ${ }^{63}$ :

1. innanzitutto, quello delle origini dell'alleanza tra Dio e Israele, collocato in un passato indefinito e, in qualche modo, «remoto» (םтק: v. 2a; cf v. 20a);

2. ai vv. $3 \mathrm{~b}-8$, la memoria riguarda un passato recente, che viene rievocato attraverso la descrizione di quanto avvenuto e il ripetuto invito a «considerare» tali avvenimenti (cf זכר e שכר ai vv. 18.22.23 e il v. 10);

3. ai vv. 12-17, infine, la memoria si dirige ancora una volta verso un passato remoto (מקדם: v. 12a), anzi verso l'origine stessa del creato, celebrato come manifestazione della regalità di Dio (מלכי: v. 12a)

61 Sul nesso di questi verbi con il tema della memoria, cf. Pavan, «He Remembered», 64-71.

62 Lespressione del v. 3a sembrerebbe implicare che l'orante pensi che alla rovina del tempio non ci sia più rimedio: così tra gli altri interpretano Spieckermann, Heilsgegenwart, 126-127; Schökel - Carniti, Salmi II, 36-37. Cf. anche Hossfeld - Zenger, Psalmen 51-100, 357.

63 Nell'identificazione di questi punti vengono considerate le occorrenze dei verbi שכר e שכת e, così come quelle dell'avverbio קדם e, in generale, la struttura dei tempi verbali.

64 L'uso dell'avverbio קדם colloca sia gli avvenimenti alle origini di Israele (v. 2) che quelli all'origine del mondo (vv. 12-17) in una sorta di passato indefinito, «mitico». Tale legame è confermato, del resto, dalle già menzionate allusioni ad Es 15 contenute agli stessi vv. 12-17. Se nel caso del v. 2 Dio è invitato a ricordare esplicitamente gli avvenimenti del passato (e gli impegni presi con l'alleanza), ai vv. 12-17 l'orante si rivolge a Dio in «tu» (dopo una sorta di «riflessione» iniziale, quasi tra sé e sé al v. 12), celebrando la sua regalità in termini «mitici». In entrambi i casi troviamo una richiesta («ricorda!») formulata in modi diversi ma tra loro collegati nella trama del salmo. Sull'uso del linguaggio mitico nel Salte- 
In questo modo, la struttura temporale del salmo appare connotata, in qualche modo, dalla memoria stessa. Da una parte, l'oblio da parte di Dio rappresenta il «presente indefinito» nel quale si svolge la preghiera dellorante; dall'altra, l'orante stesso richiama Dio stesso alla sua regalità e agli impegni presi offrendo e concludendo un'alleanza con Israele. Se la memoria delle origini (קדם: vv. 2a.12a) connota soprattutto l'inizio dei primi due blocchi del salmo (vv. 1-3 e 10-12), quella degli avvenimenti «recenti» struttura in particolare il terzo ed ultimo blocco (vv. 18-23; cf anche vv. 4-8). Al di là della loro funzione retorica - muovere Dio al «ricordo», cioé a rinnovare le sue azioni salvifiche questi riferimenti "cronologici» e l'uso mirato dei lessemi della memoria costituiscono l'asse temporale su cui si snoda la preghiera dell'orante, lo «spazio cronologico», per così dire, in cui si situa l'appello e la supplica del salmo ${ }^{65}$.

\subsection{Tempio, memoria, nome}

Il tempio ${ }^{66}$ appare come il centro spaziale del salmo, l'unico suo vero riferimento «topografico» ${ }^{67}$. Questo fatto emerge in modo particolare ai vv. $2-8$, nei quali si ricordano sia la fondazione (v. 2) che la distruzione (vv. 3b-8) del santuario.

$\mathrm{Al}$ v. 2, come già menzionato, Dio è invitato a ricordare l'alleanza stretta «a suo tempo» (קדם: v. 2a) con Israele. Gli avvenimenti vengono concatenati in modo quasi asindetico, utilizzando un vocabolario mirato ${ }^{68}$ e secondo un ordine progessivo:

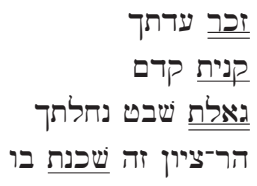

rio, cf. tra gli altri: Zenger, «Das Mythische», 233-251; Groenewald, «Mythology», 909-924; Weber, «Wasser», 261-280.

65 All'altro estremo, per contro, si colloca la speranza di un futuro intervento divino (vv. 3a.18-23), espresse dall'uso dell'imperativo e dai due jussivi del v. 21. Sulla scansione «temporale» del salmo, cf. in particolare Weiss, «Methode», 99.

66 Sul tempio, cf. in particolare: Janowski, «Himmel», 229-260; idem, «Der Ort», 369397; idem, «Die Einwohnung», 3-40.

67 Accanto a questi riferimenti vanno anche annoverate le «mitiche» designazioni spaziali dei vv. 12-17. Sullo studio della costruzione dello spazio nei salmi, cf. anche Prinsloo, «The Role of Space», 457-477; Schäder, «Understanding», 139-160.

68 Per un'enumerazione dei riferimenti intertestuali, cf. in particolare Hakham, Psalms, 14-15; Emmendörfer, Gott, 77-84. 
Il verbo principale (כפר imperativo) sostiene la struttura della frase, che prosegue con una (probabile) relativa (קנית קרם) e con una frase indipendente retta da un altro verbo (גאל qatal) ${ }^{69}$, a sua volta reggente due oggetti (הר־ציון/שבט נחלתך), il secondo dei quali è qualificato da un'ultima relativa ${ }^{70}$. La seconda proposizione sembra, in qualche modo, specificare l'affermazione più generale della prima: Dio si è acquistato una ערה nei tempi antichi, vale a dire che ha riscattato il popolo della sua eredità ${ }^{71}$, più precisamente il monte dove ha scelto di abitare (שכן). L'elezione di Israele o di una porzione del suo territorio è, così funzionale all'elezione del luogo dove abitare e il movimento del versetto procede, di conseguenza, dal generale al particolare ${ }^{72}$. Solo con il verbo שכן tale movimento trova, per così dire, riposo.

Con il v. 3, tuttavia, si introduce un improvviso contrasto: il luogo in cui Dio ha scelto di abitare è in rovina (משאות נצח: v. $3 a^{73}$ ), completamente distrutto (כל-הרע: v. 3b). I vv. 4-8 descrivono in modo drammatico come si è arrivati a questo punto. Pur nelle difficoltà di lettura presentate dai vv. 5.6, l'articolazione di questi versetti sembra snodarsi attorno alle menzioni del tempio:

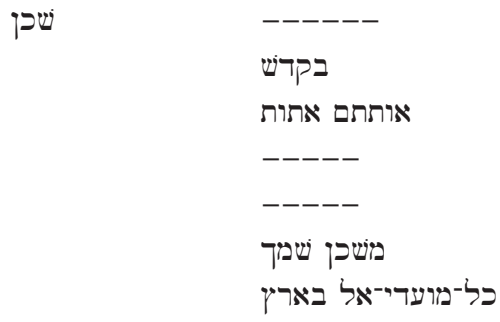

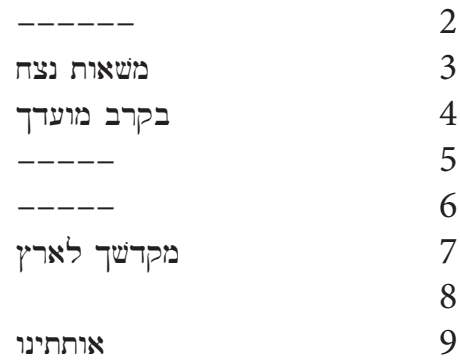

A parte la singolare espressione al v. 3a (משאות נצח), ogni denominazione è ripetuta due volte in modo variato: מקדשי/קדש (vv. 3b.7a);. משכן/שכן (vv. 2c.7b); מועדי־אל/פוערך (vv. 4a.8b); cf anche אותתינו/אותתם (vv. 4b.9a). In generale, le denominazione dei vv. 3b-8 appaiono, dunque, distribuite secondo una sorta di «scansione» fissa, con una leggera variazione:

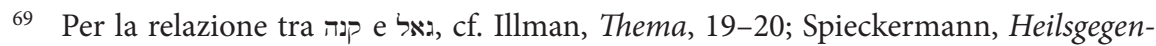
wart, 128.

70 Sul significato di in questo contesto: JM $\$ 145 \mathrm{c}$. Cf. anche Cordes, Asafpsalmen, 85;Basson, Metaphors, 206.

71 שבט נבחלתך, cf. Emmendörfer, Gott, 81-82.

72 La rappresentazione dellelezione di Israele in funzione, per così dire, del santuario si ritrova anche nel Sal 78: cf. Pavan, «He Remembered», 223-336.

73 Cf. Emmendörfer, Gott, 82-83. 


\section{קדשי}

מקדש

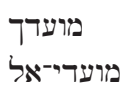

אותח

אותתינו

L'uso relativamente ampio di tali sostantivi ${ }^{74}$, per lo più associati a verbi di

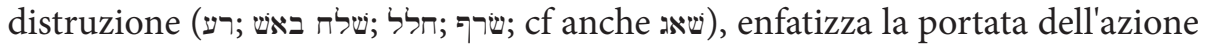
dei nemici, ritratta come un'irruzione nello spazio divino (בקרב: v. 4a) e una sua profanazione (שמו אותתם אותת: v. 4b). Tale azione è rappresentata come quella di chi distrugge come si disbosca (vv. 5-6) e di chi incendia e mette a fuoco (vv. 7-8), sottolineando, in entrambi i casi, l'aspetto «sonoro» di questa azione (cf. vv. 4a.8a; cf anche vv. 22.23) ${ }^{75}$. Se lo sviluppo dei sostantivi indicanti il tempio si «conclude» al v. 9, nella serie spicca la singolarità dellespressione משכן-שמך che riprende e amplifica il v. $2 c^{76}$.

I vv. 3b-9 si collocano, con la loro enfasi sul tempio e sul "paese» (ארץ: v. 8 b), in una sorta di relazione implicita con i vv. $12-17$, almeno da due punti di vista:

1. da una parte, i vv. 12-17 ampliano la prospettiva spaziale dei vv. 3b-9, dato che non è più solo il «paese» (ארץ: v. 8 b; cf anche vקרב: va) ma la «terra» intera (בקרב הארץ: v. 12b) ad essere considerata, come raggio di azione della signoria di Dio (cf anche v. 17) ${ }^{77}$;

2. dall'altra, i riferimenti alla regalità divina e alla sua opera di ordinatore del cosmo appaiono strettamente connessi a quelli del tempio, essendo quest'ultimo - in molti passaggi della Bibbia Ebraica e nelle letterature

74 Si nota un passaggio costante dal sostantivo in forma «semplice» (שכן) alla co-

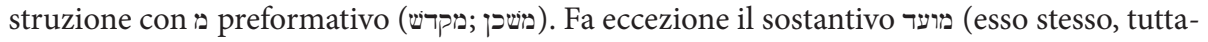

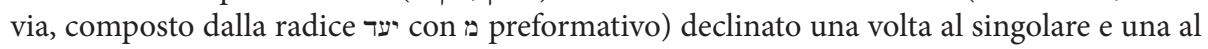
plurale. L'uso di queste tre denominazione intende rafforzare la portata di quanto descritto (una sorta di effetto retorico di «accumulo») e della richiesta soggiacente. Allo stesso tempo, rappresentano tre lessemi fondamentali per la concezione e la rappresentazione del tempio nella Bibbia Ebraica: cf. Janowski, «Der Ort», 24-68.

75 Cf. Hakham, Psalms, 16. Da qui si può forse dedurre che i verbi נאץ e (vv. 10.18) possano implicare anche un oltraggio di tipo «verbale».

76 Lespressione trova la sua radice in alcuni passi di Dt (cf. ad es. Dt 12,21; 14,23; 16,2.6.11), passaggi di cui il salmo sembra riprendere le istanze fondamentali e va probabilmente intesa in senso cultuale (il Nome come riferimento al culto liturgico). Tale riferimento, come vedremo, non è estraneo al nostro salmo. Sulle problematiche implicate in questa espressione, tra l'abbondante letteratura, cf. Reuter, Kultzentralisation; Keller, Untersuchungen; Richter, Name Theology; Otto, «Kontexte».

77 A questa dilatazione spaziale corrisponde, come visto, quella temporale. Cf. Lichtenstein, Gottesstadt, 247-255. 
del $\mathrm{VOA}^{78}$ - legato ad entrambe le realtà, quasi una loro concretizzazione spaziale.

L'allargamento di prospettiva dei vv. 12-17 istituisce, così, una sorta di contrasto con i vv. 3b-9, nel quale all'azione distruttrice dei nemici, che punta a distruggere tutto (cf כל vv. 3.8) corrisponde quella ordinatrice di Dio, che ordina tutta la terra (cf כל: v. 17). L'insistenza sui riferimenti all'ambiente acquatico dei vv. 13-15 (in una sorta di contrasto con l'immagine del fuoco ai vv. 7-8) e sui loro elementi (compresi i תנינית: vil e il 13b.14a) può alludere alle forze storiche caotiche di cui $\mathrm{i}$ «nemici» sono rappresentazione e strumento ${ }^{79}$.

Le richieste dei vv. 18-23 appaiono, da questo punto di vista, una conseguenza diretta dei precedenti o, meglio, del voluto contrasto tra i vv. 3b-9 e 1217. In questa sezione, però, non viene più menzionato il tempio e nemmeno si chiede esplicitamente la sua ricostruzione $e^{80}$ ma la supplica ruota, come visto, attorno al tentativo di «smuovere» Dio dal suo oblio perché intervenga contro i nemici (cf vv. 22.23). A creare una sorta di «ponte» con la prima parte è, però, il sostantivo שם, attorno al quale si snoda un possibile riferimento implicito al tempio nei vv. 18-23. Tale sostantivo - assente nei vv. 12-17 - ricorre quattro volte nel salmo:

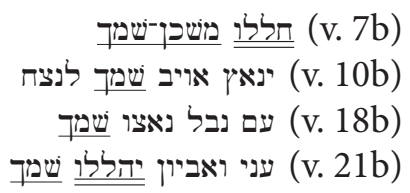

Ai vv. 10b.18b.21b il Nome (sempre con suffisso di $2^{\text {a }}$ singolare) appare in diretta relazione al v. $7 \mathrm{~b}$, in cui il tempio è definito «dimora» del Nome (משכן-שמן), espressione che si ricollega a sua volta al v. 2c, in cui il tempio come dimora divina ( $\mathrm{cf}$ al v. 2c) viene rappresentato come il culmine e lo scopo dell'elezione di Israele ${ }^{81}$. In questo modo, si comprende come la distruzione del tempio viene interpretata nei versetti chiave del salmo (vv. 10b.18b; cf anche

78 Cf. Janowski, «Himmel», 29-260.

79 Cf. Hossfeld - Zenger, Psalmen 51-100, 367-368; Greene, «Destruction», 85-101. Da questo punto di vista, i vv. 12-17 affrontano il problema della «destabilizzazione» conseguente la perdita del tempio in modo radicale, andando cioé allorigine del tempo (cf. קדם: v. 12a) e dello spazio (cf. i riferimenti spaziali ai vv. 13-17 e, in particolare, all'attività ordinatrice ai vv. 16-17) dall'attività «regale» (cf. מלכי: v. 12a) di Dio.

80 La «terra» appare, per contro, al v. 20b in preda al caos, come covo di חמלפ.

81 Cf. Janowski, «Die Einwohnung», 3-40. 
21b) come una "profanazione» del Nome, profanazione strettamente associata, come visto, anche ad una sorta di «insulto» verbale (cf anche vv. 22.23). A tale profanazione corrisponde l'auspicio che i poveri possano lodare il nome (יהללו שמך: v. 21b), espressione che può essere perciò, in qualche modo, collegata al tempio ${ }^{82}$.

Proprio il riferimento al Nome e la sottolineatura del tempio come dimora di questo permettono forse di cogliere unallusione a quest'ultimo dal punto di vista della memoria, una sorta di «spazializzazione» della memoria che l'uomo deve avere di Dio, corrispondente e speculare a quella divina ${ }^{83}$. Il sostantivo שם compare nei salmi ${ }^{84}$, infatti, in 11 casi in parallelo con il sostantivo זכ, un derivato della radice זכר il cui significato può essere quello di «nome» (divino) pronunciato ${ }^{85}$. Allo stesso modo, la costruzione הזכיר שם ricorre in diversi luoghi della Bibbia Ebraica ${ }^{86}$ per designare un atto di parola collegato al «confessare» o al "celebrare» ${ }^{87}$. In questo senso, la forma verbale זכר hifil ricorre in $1 \mathrm{Cr} 16,4$, in parallelo anche con הלל , in un passaggio in cui sono elencate le azioni che $i$ leviti cantori sono incaricati di compiere per ordine di Davide ${ }^{88}$.

Tutte queste occorrenze mettono in evidenza la possibilità che con l'espressione יהללו שמך al v. 21b si possa intendere un atto di memoria, collegato in qualche modo con culto nel tempio, dimora del Nome ${ }^{89}$. Con la perdita del tempio, Israele si ritrova, quindi, senza la possibilità di lodare il Nome e, in un

82 Questo legame può essere confermato da altri elementi. Il contrasto tra il v. 7b (חלל משכן-שמך) e il v. 21b (יהללו של שמך), giocato sulla vicinanza sonora delle radici pלל indicare due azioni antitetiche e corrispondenti. Allo stesso modo, il «rumore» dei nemici (vv. 4.23; cf. anche l'uso di ניץ e in questa accezione) e le lodi dei poveri sono, in qualche modo, poste in contrasto antitetico tra di loro. Infine, il vocabolario del v. 23 (עלה תמיר) contiene una sorprendente allusione cultuale (cf. Es 29,42; Nm 28,3.6.10.15.23.24.32; 29,6; 1 Cr 16,40; 23,31; cf. anche l'allusione cultuale nel sostantivo מוער), anche in questo caso da leggere nel senso del contrasto (al sacrificio quotidiano si è sostituito il «fragore» dei nemici). In questo senso, l'espressione del v. $21 \mathrm{~b}$ potrebbe essere letta anche come un auspicio di ricostruzione del tempio, nonostante al v. 3 le sue rovine siano state qualificate come «eterne» (נצח). Di diverso parere Spieckermann, Heilsgegenwart, 126-127.

83 Cf. in proposito Daffern, «The Psalms», 79-94.

84 Sulla presenza di questo tema nei salmi di Asaf, cf. Pavan, «He Remembered», 77-78.

85 Cf. la disanima in idem, «He Remembered», 31-74. Secondo ogni evidenza, il nome זֵֵ deriverebbe da זכר hifil.

86 Es 20,24; 3,15; cf. Is 12,4; 26,8.13; 49,1; Ger 11,9; 5; Os 2,19; Am 6,10; Zc 13,2; Sal 20,8; 45,18; 119,55; 135,13; Gb 18,17; Prv 10,7.

87 Cf. Pavan, «He Remembered», 45-49.

88 Cf. idem, «He Remembered», 192-199. Cf. anche Gl 2,26; 44,9; 69,31; 74,21; 105,3; $113,1.3 ; 135,1 .[3] ; 145,2 ; 148,5.13 ; 149,3 ; 1$ Cr 16,10; $29,13$.

89 Cf. Janowski, «Die Einwohnung», 14-18. 
certo senso, senza la possibilità di coltivare la memoria di Dio. La forma del v. 21 (due jussivi) lascia anche intendere che questa «memoria» possa pure essere il frutto del rinnovato intervento divino, secondo una corrispondenza tra memoria divina e umana esplicitata altrove nel Salterio ${ }^{90}$. Le due accezioni - tempio come luogo del Nome e della memoria e lode/memoria come frutto dell'agognato intervento divino - richiamano, quindi, il fatto che con la perdita del "centro simbolico» del tempio Israele vede messa in pericolo la sua "memoria» e, di fatto, la sua stessa esistenza.

\section{Conclusione}

La struttura spazio-temporale del Sal 74, tracciata attraverso i riferimenti alla memoria e al tempio, mette in luce come l'orante - individuale e/o collettivo si trovi in una condizione di «de-localizzazione» o spaesamento e di immersione in una sorta di "eterno presente». Alla prima appartiene soprattutto la distruzione e la profanazione (חלל: v. 7b) della dimora del Nome, il cui effetto è la mancanza di un centro spaziale e simbolico in cui sia possibile lodarlo (cf הלל al v. 21b) (19 $^{91}$. Alla seconda appartiene, invece, l'insistenza sulla durata eterna dell'ira divina (לנצח: vv. 1.10.17; cf v. 3) e dell'oltraggio dei nemici (cf al v. 23), meglio qualificati - attraverso l'insistenza degli «imperativi della memoria» (vv. 2.18.19.22.23) - come oblio divino. Al cuore o all'incrocio di entrambe le realtà si colloca il Nome divino, in relazione al quale si configurano lo spazio e il tempo e a causa della «perdita» del quale l'orante si percepisce in una condizione di oblio ${ }^{92}$.

Se i riferimenti alla memoria e loblio permettono di strutturare la percezione dello spazio e del tempo all'interno del salmo come decentramento/spaesamento e come «immobilità», la stessa realtà (memoria) permette di individuare anche la «via di uscita» percorsa dall'orante in tale situazione. Di particolare importanza appaiono, in proposito, i riferimenti al passato evidenziati nell'analisi (vv. 2.3-9.12-17) e, tra questi, l'inno dei vv. 12-17: quest'ultimo, infatti, appare

90 Cf. Pavan, «He Remembered», 31-74.

91 Lapplicazione di questa categoria allo studio dei salmi non è nuova: cf. Brueggemann, «Psalms», 3-32.

92 Come detto, questo oblio può anche essere inteso dal versante «umano», per così dire: con la distruzione del tempio, viene meno il principale «luogo della memoria» di Dio, là dove Israele perpetua il ricordo del Signore attraverso la lode liturgica. Cf. Pavan, «He Remembered», 31-74. 
una sorta di «memoria in atto» ${ }^{93}$, il cui scopo è quello di fondare le richieste dei vv. 18-23 attraverso un "riorientamento» verso l'origine di ogni atto salvifico, un riorientamento che, in un certo senso, anticipa e prelude alla lode auspicata e invocata (v. 22b) ${ }^{94}$. Tale riorientamento si mostra in grado, in un certo senso, di spezzare il cerchio dell' «eterno presente», anche se, in ultima analisi, l'esito della preghiera rimane incerto: ai vv. 18-23 l'orante non invoca direttamente la ricostruzione del tempio e le espressioni che vengono usate rimangono aperte a diverse interpretazioni ${ }^{95}$.

\section{Bibliografia}

Auffret, P., «Essai sur la structure litteraire du Psaume LXXIV», VT 33 (1983) 129-148. Auffret, P., «"Souviens-toi de ton assemblée!”. Étude structurelle du Psaume 74», FoOr (1997), 21-31.

Barbiero, G., Salmi scelti dal terzo libro del salterio (Dispense ad uso degli uditori. Pontificio Istituto Biblico; Roma 2007).

Basson, A., Divine Metaphors in Selected Hebrew Psalms of Lamentation (FAT II.15; Tübingen 2006).

Basson, A., «"Only Ruins Remains”. Psalm 74 as a Case of Mundus Inversus», OTE (2007), 128-137.

Baumann, G., «Psalm 74: Myth as the Source of Hope in Times of Devastation», D.J. Human (ed.), Psalms and Mythology (LHB.OTS 462; New York 2007), 91-103.

Begg, C.T., «The Covenantal Dove in Psalm LXXIV 19-20», VT 37 (1987), 78-81.

Boadt, L., "The Use of "Panels" in the Structure of Psalms 73-78», CBQ 66 (2004), 533-550.

Bouzard, W.C., We Have Heard with Our Ears, O God. Sources of the Communal Laments in the Psalms (SBL.DS 159; Atlanta, GA 1997).

Brown, W.P., Seeing the Psalms. A Theology of Metaphor (Louisville, KY 2002).

Broyles, C.L., The Conflict of Faith and Experience in the Psalms. A Form-Critical and Theological Study (JSOT.S 52; Sheffield 1989).

Brueggemann, W., «The Psalms and the Life of Faith: a Suggested Typology of Function», JSOT 17 (1980), 3-32.

93 Riprendiamo qui le osservazioni formulate per Sal 77,14-21, testo per tanti versi simile a Sal 74,12-17: cf. idem, «Memoria», 69-90.

94 Cf. Baumann, «Psalm 74», 91-103.

95 Cf. Weiss, «Methode», 103-106. Non è possibile in questa sede sviluppare la prospettiva di lettura qui proposta e le sue implicazioni tenendo conto del contesto canonico del Sal 74 (Sal 73-89). In questo senso, cf. le osservazioni di Cole, Shape, 28-30; Hossfeld Zenger, Psalmen 51-100, 371; Boadt, «Panels», 541; Barbiero, Terzo libro, 42-43; Pavan, «He Remembered», 75-222. Cf. anche Jensen, «Psalm 75», 419. 
Castellino, G.R., Libro dei Salmi (SB; Torino 1955).

Clines, D.J.A., The Dictionary of Classical Hebrew. Volume III. ז-ט (Sheffield 1996).

Cole, R., The Shape and Messagge of Book III (Psalms 73-89) (JSOT.S 307; Sheffield 2000).

Cordes, A., Die Asafpsalmen in der Septuaginta. Der griechische Psalter als Übersetzung und theologisches Zeugnis (HBS 41; Freiburg i.B. 2004).

Daffern, M., «The Psalms: Places for Remembering», J. Flebbe (ed.), Holy Places in Biblical and Extrabiblical Traditions (Göttingen 2016), 79-94.

Dahood, M., Psalms. II: 51-100 (AB 17; Garden City, NY 1968).

Donner, H., «Argumente zur Datierung des 74. Psalm», J. Schreiner (ed.), Wort, Lied und Gottesspruch. Beiträge zur Septuagint. FS für Jospeh Ziegler. Vol. I (FzB 2; Würzburg 1972), 41-50.

Emerton, J.A., «Notes on Three Passages in Psalms Book III», JThS 14 (1963), 374-381.

Emerton, J.A., «Translation of Psalm 74:4», JTS (1976), 391-392.

Emmendörfer, M., Der ferne Gott. Eine Untersuchung der alttestamentlichen Volksklagelieder vor dem Hintergrund der mesopotamischen Literatur (FAT 21; Tübingen 1998).

Engle, R.W., Psalm 74. Studies in Content, Structure, Context, and Meaning (Grace Theological Seminary Diss.; Winona Lake 1987).

Fishbane, M., «The Sacred Center: the Symbolic Structure of the Bible», M. Fishbane, P.R. Flohr (edd.), Texts and Responses. FS N.N. Glatzer (Leiden 1975), 6-27.

Gärtner, J., Die Geschichtspsalmen. Eine Studie zu den Psalmen 78, 105, 106, 135 und 136 als hermeneutische Schlüsseltexte im Psalter (FAT 84; Tübingen 2012).

Gerstenberger, E.S., Psalms. Part 2 and Lamentations (FOTL 15; Grand Rapids, MI 2001).

Girard, M. (ed.), Les Psaumes redécouverts (Montréal 1994-1996).

Goldingay, J., Psalms. Volume 2: Psalms 42-89 (Grand Rapids, MI 2007).

Greene, N.E., «Destruction, and a Psalmist's Plea: Rethinking the Poetic Structure of Psalm 74», JBL (2017), 85-101.

Groenewald, A., «Mythology, Poetry and Theology», HTS (2006), 909-924.

Hakham, A., The Book of Psalms. II (Jerusalem 1981) [Ebr.].

Hartenstein, F., Die Unzugänglichkeit Gottes im Heiligtum. Jesaja 6 und der Wohnort JHWHs in der Jerusalemer Kulttradition (WMANT 75; Neukirchen-Vluyn 1997).

Hossfeld, F.-L., Zenger, E., Psalmen 51-100 (HThK.AT; Freiburg i.B.-Basel-Wien 2000).

Human, D., «Berit in Psalm 74», Skrif en Kerk (1995), 57-66.

Illman, K.J., Thema und Tradition in den Asaf-Psalmen (Åbo 1976 1976).

Janowski, B., «Der Himmel auf Erden. Zur kosmologischen Bedeutung des Tempels in der Umwelt Israels», B. Janowski, B. Ego (edd.), Das biblische Weltbild und seine altorientalischen Kontexte (FAT 32; Tübingen 2001), 229-260.

Janowski, B., «Die heilige Wohnung des Höchsten. Kosmologische Implikationen der Jerusalemer Tempeltheologie», O. Keel, E. Zenger (edd.), Gottesstadt und Got- 
tesgarten. Zu Geschichte und Theologie des Jerusalemer Tempels (QD 191; Freiburg 2002), 24-68.

Janowski, B., «Das Doppegesicht der Zeit. Alttestamentliche Variationen zum Thema "Mythos und Geschichte"», B. Janowski (ed.), Die Welt als Schöpfung. Beiträge zur Theologie des Alten Testaments (Neukirchen-Vluyn 2008), 79-104.

Janowski, B., «Der Ort des Lebens. Zur Kultsymbolik des Jerusalemer Tempels», J. Kamlah (ed.), Temple Building and Temple Cult. Architecture and Cultic Paraphernalia of Temples in the Levant (2.-1. Mill. B.C.E.) (Abhandlungen des Deutschen Palätina-Vereins 41; Wiesbaden 2012), 369-397.

Janowski, B., «Die Einwohnung Gotts in Israel. Eine religions- und theologiegeschichtliche Skizze zur biblischen Schekina-Theologie», B. Janowski, E.E. Popkes (edd.), Das Geheimnis der Gegenwart Gottes (WUNT 318; Neukirchen-Vluyn 2014), 3-40.

Jensen, J.E., «Psalm 75: Its Poetic Context and Structure», CBQ 63 (2001), 416-429.

Keller, M., Untersuchungen zur deuteronomisch-deuteronomistischen Namestheologie (BBB 105; Weinheim 1996).

Körting, C., Zion in den Psalmen (FAT 48; Tübingen 2006).

Lichtenstein, M., Von der Mitte der Gottesstadt bis ans Ende der Welt. Psalm 46 und die Kosmologie der Zionstradition (WMANT 139; Neukirchen-Vluyn 2014).

Lippke, F., «„Thou Driest Up Mighty Rivers” (Ps 74:15). History of the Text and Archaelogical Implications», M. Augustin, H.M. Niemann (edd.), "My Spirit at Rest in the North Country» (Zecariah 6.8). Collected Communications to the XXth Congress of the International Organization for the Study of the Old Testament, Helsinki 2010 (BEATAJ 57; Frankfurt a. M. 2011), 141-152.

Loretz, O., Leberschau, Sündenbock, Asasel in Ugarit und Israel. Leberschau und Jahwestatue in Psalm 27, Leberschau in Psalm 74 (UBL 3; Altenberge 1985).

Lugt, P.v.d., Cantos and Strophes in Biblical Hebrew Poetry. With Special Reference to the First Book of the Psalter (OTS 53; Leiden-Boston 2006).

Lugt, P.v.d., Cantos and Strophes in Biblical Hebrew Poetry. II: Psalms 42-89 (OTS 57; Leiden-Boston 2010).

Otto, E., «Altorientalische Kontexte der deuteronomischen Namenstheologie», ZAR (2007), 237-248.

Pavan, M., «Il “nemico” nel III libro del salterio (Sal 73-83)», RivBib 57 (2009), 273-298.

Pavan, M., «La memoria nel Sal 77», RivBib 60 (2012), 69-90.

Pavan, M., «He Remembered That They Were But Flesh, a Breath That Passes and Does Not Return» (Psalm 78,39). The Theme of Memory and Forgetting in the Third Book of the Psalter (Psalm 73-89) (ÖBS 44; Frankfurt a.M. 2014).

Petrany, C., Pedagogy, Prayer, and Praise. The Wisdom of the Psalms and Psalter (FAT. II 83; Tübingen 2015).

Ploeg, J.P.M.v.d., «Psalm 74 and Its Structure», M.S.H.G.H.v. Voos, P.H.J.H.t. Cate, N.A.v. Uchelen (edd.), Travels in the World of the Old Testament. Studies Presented to M.A. Beek on the Occasion of His 65th Birthday (SSN 16; Assen 1974), 204-210.

Prinsloo, G.T.M., «The Role of Space [...] (Psalms 120-134)», Bib. (2005), 457-477. 
Reuter, E., Kultzentralisation. Entstehung und Theologie von Dt 12 (BBB 87; Frankfurt a.M. 1993).

Richter, S.L., The Deuteronomistic History and the Name Theology. Lešakkēn šemô šām in the Bible and the Ancient Near East (BZAW 318; Berlin-New York 2002).

Robinson, A., «A Possible Solution to the Problems of Psalm 74:5», ZAW (1967), 120 121.

Schäder, J.-M., «Understanding (the lack of) Space in Psalm 47:6 in Light of Its Neighbouring Psalms: A Spatial Reading of Psalms 46-48», OTE (2010), 139160.

Schökel, L.A., Carniti, C., I Salmi (Roma 1993).

Sollamo, R., "The Simile in Ps 74:5: A Wood-Cutter Entering a Forest Wielding Axes», Svensk Exegetisk Årsbok (1989), 178-187.

Spieckermann, H., Heilsgegenwart. Eine Theologie der Psalmen (FRLANT 148; Göttingen 1989).

Tate, M.E., Psalms 51-100 (WBC 20; Dallas, TX 1990).

Tsumura, D.T., «The Creation Motif in Psalm 74:12-14? A Reappraisal of the Theory of the Dragon Myth», JBL (2015), 547-555.

Venter, P.P., «Salvation for Earth? A Body Critical Analysis of Psalm 74», OTE (2008), 533-545.

Wagner, A., «Dichten und Denken. Zum Verständnis des "Personenwechsel” in alttestamentlicher, ugaritischer und verwandter Literatur», M. Kropp, A. Wagner (edd.), Schnittpunkt Ugarit (Frankfurt a.M. 1999), 271-284.

Wagner, A., Beten und Bekennen. Über Psalmen (Neukirchen-Vluyn 2008).

Wagner, A., «Strukturen des Gebets im Alten Testament», M. Witte, J.F. Diehl (edd.), Orakel und Gebete. Interdisziplinäre Studien zur Sprache der Religion in Ägypten, Vorderasien und Griechenland in hellenistischer Zeit (FAT II.38; Tübingen 2009), 197-216.

Wälchli, S.H., Gottes Zorn in den Psalmen. Eine Studie zur Rede vom Zorn Gottes in den Psalmen im Kontext des Alten Testaments und des Alten Orients (OBO 244; Göttingen 2012).

Weber, B., “"Es sahen dich die Wasser - sie bebten...” (Ps 77:17b): die Funktion mytho- poetischer Sprache im Kontext von Psalm 77», OTEs 19 (2007), 261-280.

Weber, B., “"Es gibt keine Rettung für ihn bei Gott!” (Psalm 3,3). Direkte Rede von und an "Widersacher(n)" in den Psalmen», A. Ruwe (ed.), Due bist es, ein Mensch meinsgleichen (Psalm 55,14). Ein Gespräch über Psalm 55 und seine Parallelen (Biblisch-Theologisch Studien 157; Neukirchen-Vluyn 2016), 191-267.

Weber, B., Werkbuch Psalmen III. Die Psalmen 73 bis 150 (Stuttgart 2016).

Weiss, M., «Die Methode der «Total-Interpretation»», Congress Volume Uppsala 1971 (VT.S 22; Leiden 1972), 88-112.

Willesen, F., «The Cultic Situation of Psalm LXXIV», VT 11 (1952), 289-306. 
Zenger, E., «Das Mythische in den Psalmen 84 und 85», A. Lange, H. Lichtenberger, D. Römheld (edd.), Mythos im Alten Testament und seiner Umwelt. FS H.-P. Müller (BZAW 278; Berlin-New York 1999), 233-251.

Zenger, E., "IIch liebe den Ort, da deine Herrlichkeit wohnt" (Ps 26,8). Tempeltheologische Semiotiserung des Alltags im Psalter», O. Keel - E. Zenger (edd.), Gottesstadt und Gottesgarten. Zu Geschichte und Theologie des Jerusalemer Tempels (QD 191; Freiburg i.B. 2002), 180-206. 